\author{
Federico Mainardi \\ Ferdinardo Maggioni \\ Filippo Dainese \\ Giorgio Zanchin
}

\section{Development of a ICHD-II based computerised system for the general practitioner}

Published online: 20 July 2005

F. Mainardi (西) • F. Maggioni • F. Dainese G. Zanchin

Headache Centre,

Department of Neurosciences,

University of Padua, Padua, Italy

e-mail: giorgio.zanchin@unipd.it

Tel.: +39-049-8211702

Fax: +39-049-8272335

\begin{abstract}
We present a computerised programme designed for use in the office of a general practitioner. The system provides an assisted diagnosis according to the ICHD-II criteria for the principal forms of primary headaches (migraine, tension-type headache, cluster headache) and highlights the red flags of a possible secondary headache. A relevant feature is that explanations for the selection of a particular diagnosis are given at the end of the process; furthermore, the characteristics of the patient's headache, which were previ-
\end{abstract}

ously inserted in the programme by the physician, are summarised, allowing critical evaluation of the suggested diagnosis. The software can also be used as a clinical file, in that it is possible to create for each patient a clinical chart in which to record the selected diagnosis, the recommended therapy and any eventual comments. Our programme aims for educational growth, promoting the learning of the basic ICHD-II criteria.

Key words Diagnosis • Software • International Headache Classification

\section{Introduction}

In the clinical setting of a general practitioner (GP), migraine represents the most frequently observed neurological disorder [1], a point that reflects its distribution in the population. The prevalence of this primary headache ranges between $6 \%$ and $12 \%$ in males and between $15 \%$ and $18 \%$ in females, reaching a peak between the second and fourth decades of life, when productivity is greater, thus causing considerable social and economic costs [2]. In the absence of pathognomonic neuroimaging or laboratory tests, the recently revised diagnostic criteria (International Classification of Headache Disorders, 2nd edition, ICHDII) [3] are the backbone of the diagnosis of migraine, which is based on an accurate medical history centred on the clinical characteristics of headache, and on the general physical and neurological examinations; further verification is required when an underlying organic pathology is suspected of being responsible for the headache. The ICDH-II classification is structured in a ranked hierarchy: a code identifies each type of headache (the first digit corresponds to the first diagnostic level); a more detailed diagnosis entails the addition of other digits, corresponding to the more thorough diagnostic levels for a particular headache type, allowing its use not only in daily clinical practice but also in Headache Centres, or for research.

Unexpectedly, given the disability of the migraine attack (e.g., cancelled work and social activities, limitations in the care of the family, reduced work productivity), a high percentage of patients (30\%-70\% according to different studies) has never undergone a specialised medical visit [4]; among the principal causes given by the patients themselves is the belief in the inefficacy of therapy. In the 
case of medical consultation, the professional figure who was first consulted by more than $40 \%$ of migraineurs was the GP [5]. The workload of GPs obliges them to optimise the length of the visit, thus making it often difficult to properly diagnose the headache, which per se requires that adequate time be dedicated to each patient. The consequence of a too quick approach to the migraine sufferer can prevent a precise diagnosis and limit the quality of the therapeutic choices.

Knowledge of the ICHD-II criteria for the diagnosis of headaches [3], and in particular of the more frequent primary headaches, becomes indispensable for a correct diagnostic approach, preliminary to the choice of the most appropriate therapy for that particular form of headache. However, maintaining medical competency at its best is very difficult in a time characterised by the exponential increase of knowledge in this field, even more so in a setting such as that of the GPs, who must meet the diverse needs of their patients in very different areas. For this reason, we developed a computerised programme designed for use in the office of a GP, which completely revisits a previous version: the system provides an assisted diagnosis according to ICHD-II criteria for the principal forms of primary headaches (migraine, tension-type headache, cluster headache) and highlights the red flags of a possible secondary headache. A relevant feature is that explanations for the selection of a particular diagnosis are given at the end of the process. Furthermore, the characteristics of the patient's headache, which were previously inserted in the programme by the physician, are summarised, allowing critical evaluation of the suggested diagnosis. Completion of the diagnostic workup is simplified by a system of windows containing informative notes and explanations of the terminology used, which is indicated by an appropriate symbol; moving the pointer of the mouse over it opens the window with the comments and related notes. If the presenting clinical picture bears anomalies or is unusual, the programme advises the interviewer to request an in-depth visit by a specialist; the same advice, this time with the diagnosis, is provided if the characteristics of the headache are compatible with a rare form of primary headache (for example SUNCT syndrome).

The software can also be used as a clinical file, in that it is possible to create for each patient a clinical chart in which to record the selected diagnosis, the recommended therapy and any eventual comments; the clinical chart can be updated at each successive follow-up. Therefore, once the procedure has become familiar, our programme is also a valuable time-saving tool.

This programme accomplishes a twofold objective. Firstly, it provides the GP with support during the diagnostic evaluation, offering also a didactic content consisting of explanations and summary notes that appear along the steps of the programme and with the final diagnosis. Secondly - and this, together with the simplicity of the programme interface, represents the most interesting feature of this software - our programme is not a passive diagnostic instrument producing more or less "automatic" diagnostic labels, but instead constitutes aims for educational growth, promoting the learning of the basic ICHD-II criteria, and hence the critical attitude of the GP in his clinical approach to the headache patient.

\section{References}

1. MacDonald BK, Cockerell OC, Sander JW, Shorvon SD (2000) The incidence and lifetime prevalence of neurological disorders in a prospective community-based study in the UK. Brain 123:665-676
2. Zanchin G, Mainardi F, Dainese F, Maggioni F (2002) Epidemiologia delle cefalee. In: Gallai V, Pini LA (eds) Trattato delle cefalee. Centro Scientifico Editore, Torino, pp 17-33

3. Headache Classification Subcommittee of the International Headache Society (2004) The International Classification of Headache Disorders, 2nd edn. Cephalalgia 24[Suppl 1]:9-160
4. MacGregor A (1997) The doctor and the migraine patient: improving compliance. Neurology 48[Suppl 3]:16-20

5. Lipton RB, Stewart WF, Simon D (1998) Medical consultation for migraine: results from the American Migraine Study. Headache 38:87-96 